\title{
Subpicosecond Fiber Optical Parametric Chirped Pulse Amplifier Based On Highly-Nonlinear Fiber
}

\author{
Yue Zhou, Qin Li, Kim K. Y. Cheung, Sigang Yang, P. C. Chui, and Kenneth K. Y. Wong* \\ Photonic Systems Research Laboratory, Department of Electrical and Electronic Engineering, \\ The University of Hong Kong, Pokfulam Road, Hong Kong. \\ *E-mail: kywong@eee.hku.hk
}

\begin{abstract}
We experimentally demonstrate a fiber optical parametric chirped pulse amplifier. A 750 -fs signal is stretched to $40 \mathrm{ps}$, amplified with a gain of $30 \mathrm{~dB}$ through parametric process and then compressed to $808 \mathrm{fs}$.

2010 Optical Society of America

OCIS codes: (060.4370) Nonlinear optics, fibers; (190.4970) Parametric oscillators and amplifiers.
\end{abstract}

\section{Introduction}

Optical parametric chirped pulse amplification (OPCPA) has been investigated comprehensively [1] and is recognized as a key technique to amplify ultrafast pulses. Using OPCPA configuration, people can achieve extremely high peak power pulses [2]. OPCPA has some advantages over other CPA techniques, such as a broad bandwidth, good thermal properties, and access to arbitrary wavelength ranges [1]. OPCPA in optical fibers, which is called fiber optical parametric chirped pulse amplification (FOPCPA), has been proposed and numerical simulated by Hanna et al. [3]. Comparing with conventional OPCPA systems based on $\chi^{(2)}$ nonlinear effect of crystals, FOPCPA based on $\chi^{(3)}$ nonlinear effect of optical fibers allows a longer interaction length, eliminates the need for alignment and offers further integration with other fiber components.

In this paper, a FOPCPA is experimentally demonstrated. We use a 750-fs pulse at $1595 \mathrm{~nm}$ as the input signal of the FOPCPA system. The peak power of the signal is amplified from $93 \mathrm{~mW}$ to $10 \mathrm{~W}$. The proof-of-concept demonstration of FOPCPA may lead to further development of the OPCPA system. The fiber-integrated nature of the whole system allows complete self-alignment and further integration to other fiber-based systems.

2. Experimental Setup

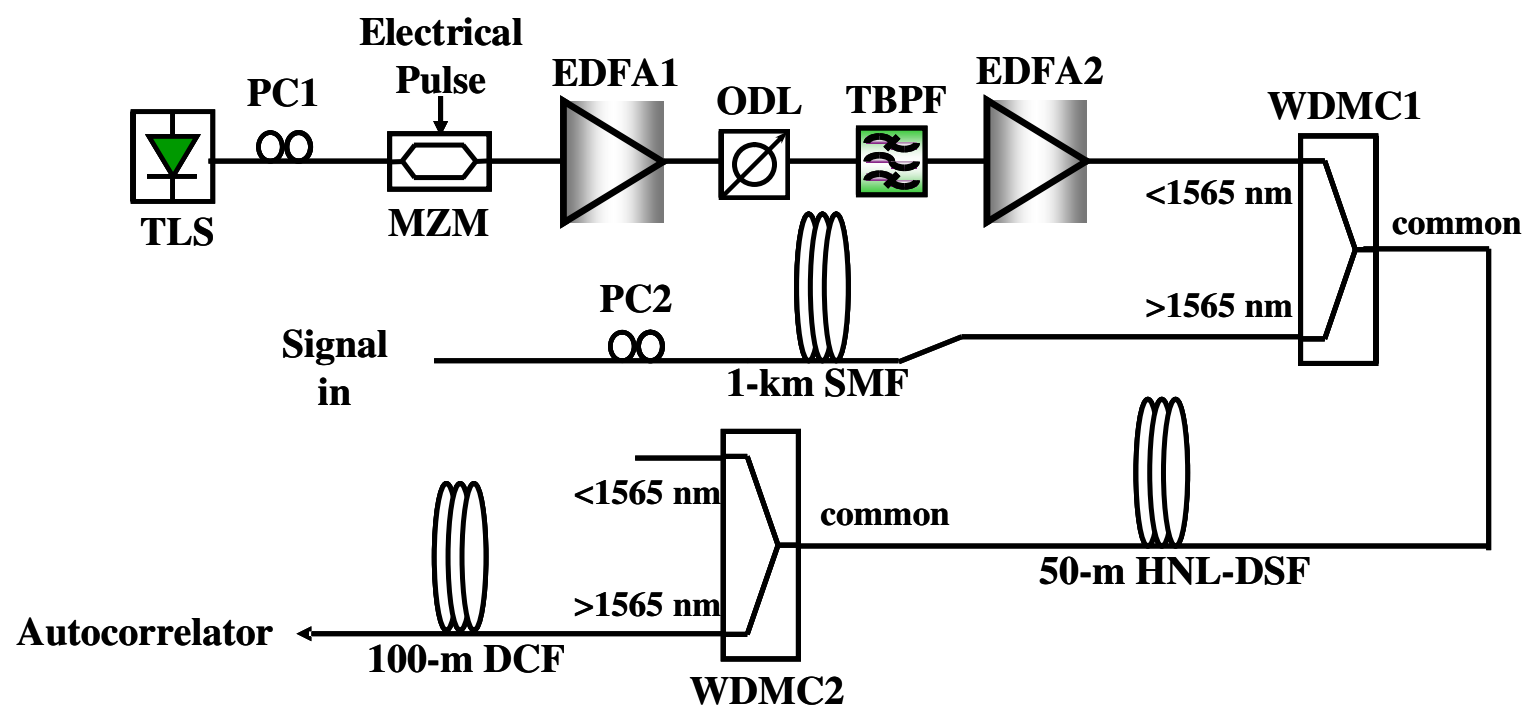

Fig. 1. Experimental setup of the FOPCPA. EDFA: erbium-doped fiber amplifier, TBPF: tunable band-pass filter, MZM: Mach-Zehnder modulator, HNL-DSF: highly-nonlinear dispersion-shifted fiber, WDMC: wavelength-division multiplexing coupler, ODL: optical delay line.

The experimental setup of the FOPCPA is shown in Fig. 1. The signal we chose had a center wavelength of $1595 \mathrm{~nm}$, pulsewidth of $750 \mathrm{fs}$ and linewidth of $5 \mathrm{~nm}$. Polarization controller PC2 was used to align the state of polarization (SOP) of the signal with that of the pump so as to maximize the parametric gain. A spool of 1-km single-mode fiber (SMF) with a dispersion of $20 \mathrm{ps} / \mathrm{nm} / \mathrm{km}$ at $1595 \mathrm{~nm}$ was used to stretch the signal from 750 fs to $40 \mathrm{ps,} \mathrm{with} \mathrm{a}$ stretching ratio of larger than 50. The peak power of the signal decreased from $93 \mathrm{~mW}$ to $1.5 \mathrm{~mW}$ after the stretcher. The pump source was a tunable laser source (TLS), which was fixed at $1555 \mathrm{~nm}$. The continuous-wave (CW) output of 


\section{CTuM1.pdf}

the TLS was intensity-modulated by a 100-MHz electrical pulse with duty ratio of $1 / 100$ to produce optical pump pulse with pulsewidth of $100 \mathrm{ps}$ and repetition rate of $100 \mathrm{MHz}$. It was then amplified by EDFA1 and EDFA2 and filtered by the TBPF with a linewidth of 1-nm to produce low-noise, high power pump. The ODL was used to synchronize the pump with the signal. The pump and signal were combined by WDMC1 with a separation wavelength at $1565 \mathrm{~nm}$ and launched into a 50-m HNL-DSF which had nonlinear coefficient of $14 \mathrm{~W}^{1} \mathrm{~km}^{-1}$, zero-dispersion wavelength of $1554.7 \mathrm{~nm}$ and dispersion slope of $0.035 \mathrm{ps} / \mathrm{nm}^{2} / \mathrm{km}$ for parametric process. After parametric amplification, the signal was filtered by WDMC2, and compressed by a spool of 100-m dispersion compensation fiber (DCF) with a dispersion of $-96.6 \mathrm{ps} / \mathrm{nm} / \mathrm{km}$ at $1595 \mathrm{~nm}$ to achieve a high peak power. The pulsewidth of the compressed signal was measured using an autocorrelator.

\section{Results and Discussions}
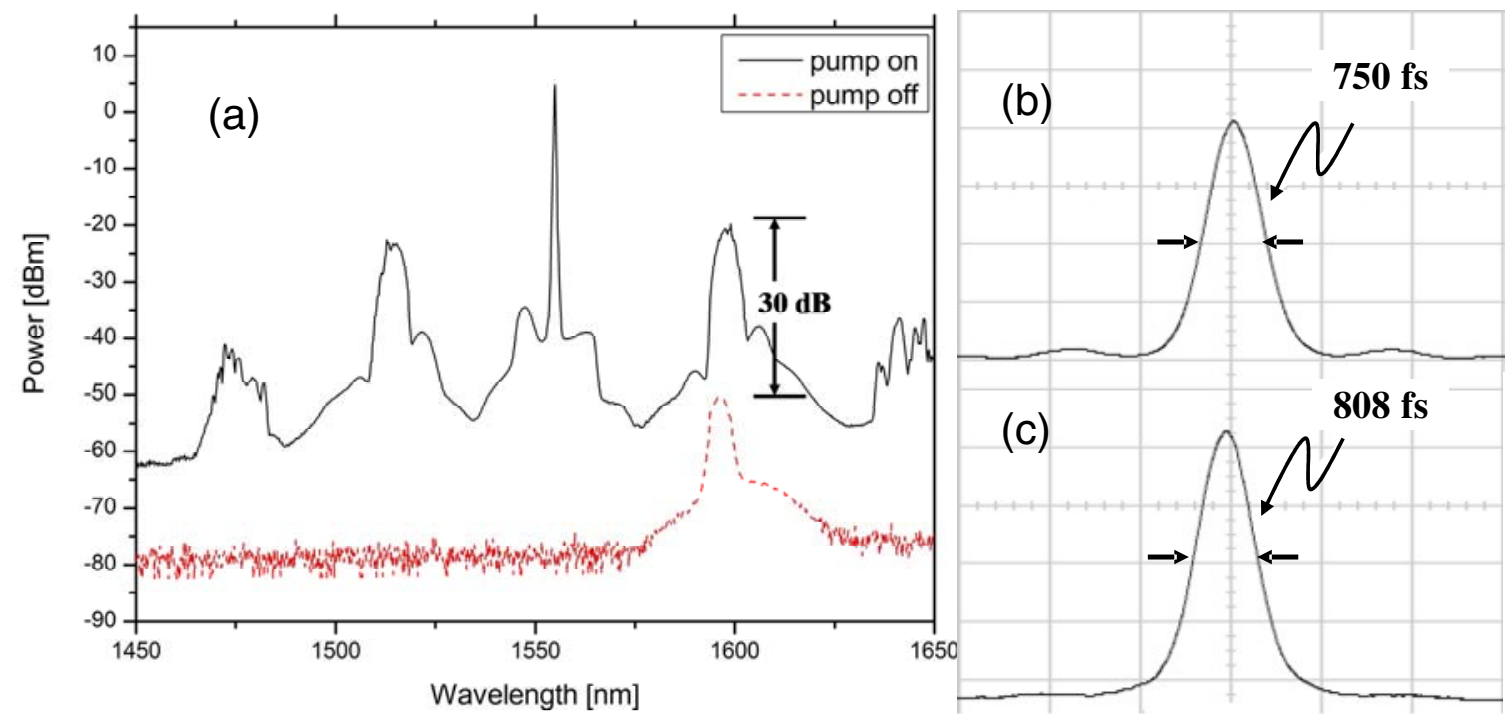

Fig. 2. (a) Optical spectra measured at HNL-DSF output when the pump is switched off (red dashed line) and on (black solid line); (b) autocorrelation trace of the original signal pulse and (c) autocorrelation trace of the amplified signal after compression.

Fig. 2 (a) shows the signal spectra measured at the HNL-DSF output when the pump is switched off (red dashed line) and on (black solid line). It can be observed that the signal receives a parametric gain of $30 \mathrm{~dB}$, and a strong idler at $1514 \mathrm{~nm}$ is also generated. The smaller peaks are due to the spurious four-wave mixing (FWM). The pedestal at the bottom of the pump is due to the amplified spontaneous emission (ASE) noise from the EDFA2. The peak power of the signal pulse after HNL-DSF is measured to be $1 \mathrm{~W}$.

Fig. 2 shows the autocorrelation traces of the original signal pulse (b) and the amplified signal pulse after compression (c). The amplified signal, which has a pulsewidth of $40 \mathrm{ps}$ and peak power of $1 \mathrm{~W}$, is compressed by the DCF. The compressed pulse has a peak power of $10 \mathrm{~W}$, and a pulsewidth of $808 \mathrm{fs}$, slightly wider than the original corresponding signal pulse. The wider pulsewidth is possibly due to residual high-order dispersion of the compressor.

\section{Conclusion}

In conclusion, we demonstrated a FOPCPA to amplify a subpicosecond signal at $1595 \mathrm{~nm}$. The 750 -fs signal was stretched to $40 \mathrm{ps}$, amplified by an all-fiber optical parametric amplifier and then compressed to 808 fs. The peak power of the signal was amplified from $93 \mathrm{~mW}$ to $10 \mathrm{~W}$. This technique has potential applications in amplification of ultrafast pulses in non-conventional wavelength bands.

\section{Acknowledgment}

The work described in this paper was partially supported by grants from the Research Grants Council of the Hong Kong Special Administrative Region, China (Project No. HKU 7179/08E and HKU 7183/09E). The authors would also like to acknowledge Sumitomo Electric Industries for providing the HNL-DSF.

\section{References}

[1] A. Dubietis, R. Butkus, and A. Piskarskas, IEEE J. Select. Topics. Quant. Electron. 12, 163-172 (2006).

[2] X. Yang, Z. Xu, Y. Leng, H. Lu, L. Lin, Z. Zhang, R. Li, W. Zhang, D. Yin, and B. Tang, Opt. Lett. 27, 1135-1137 (2002).

[3] M. Hanna, F. Druon, and P. Georges, Opt. Express, 14, 27832790 (2006). 